\begin{tabular}{|c|l|}
\hline Title & $\begin{array}{l}\text { A nalysis of the structure and neuritogenic activity of chondroitin sulfate/dermatan sulfate hybrid chains from porcine } \\
\text { fetal membranes }\end{array}$ \\
\hline Author(s) & Hashiguchi, Taishi; Mizumoto, Shuji; Y amada, Shuhei; Sugahara, Kazuy uki \\
\hline Citation & $\begin{array}{l}\text { Glycoconjugate Journal, 27(1), 49-60 } \\
\text { https://doi.org/10.1007/310719-009-9253-x }\end{array}$ \\
\hline Issue Date & 2010-01 \\
\hline Doc URL & http://hdl.handle.net/2115/49186 \\
\hline Rights & The final publication is available at www.springerlink.com \\
\hline Type & article (author version) \\
\hline File Information & GJ27-1_49-60.pdf \\
\hline
\end{tabular}

Instructions for use 


\title{
Analysis of the Structure and Neuritogenic Activity of Chondroitin Sulfate/Dermatan Sulfate Hybrid Chains from Porcine Fetal Membranes
}

\author{
Taishi Hashiguchi, Shuji Mizumoto, Shuhei Yamada, and Kazuyuki Sugahara
}

\begin{abstract}
The amniotic membrane (AM) is the innermost layer of fetal membranes and possesses various biological activities. Although the mechanism underlying these biological activities remains unclear, unique components seem to be involved. AM contains various extracellular matrix components such as type I collagen, laminin, fibronectin, hyaluronic acid, and proteoglycans bearing chondroitin sulfate/dermatan sulfate (CS/DS) glycosaminoglycan side chains. To elucidate the function of CS/DS in $\mathrm{AM}$, the structure and bioactivity of the CS/DS chains from porcine fetal membranes (FM-CS/DS) were investigated. A compositional analysis using various chondroitinases revealed that the characteristic DS domain comprised of iduronic acid-containing disaccharide units is embedded in FM-CS/DS, along with predominant disaccharide units, GlcA-GalNAc, GlcA-GalNAc(4-O-sulfate), and GlcA-GalNAc (6-O-sulfate), where GlcA and GalNAc represent D-glucuronic acid and $N$-acetyl-D-galactosamine, respectively. The average molecular size of FM-CS/DS chains was unusually large and estimated to be $250-300 \mathrm{kDa}$. The FM-CS/DS chains showed neurite
\end{abstract}


outgrowth-promoting activity with a dendrite-like morphology, which was eliminated by digestion with chondroitinase $\mathrm{ABC}$ of the CS/DS chains. This activity was suppressed by antibodies against growth factors including pleiotrophin, midkine, and fibroblast growth factor-2. The binding of these growth factors to FM-CS/DS was also demonstrated by surface plasmon resonance spectroscopy.

Keywords Chondroitin sulfate, Dermatan sulfate, L-Iduronic acid, Amniotic membrane, Fetal membranes, Neurite outgrowth-promoting activity.

\begin{abstract}
Abbreviations
GAG, glycosaminoglycan; CS, chondroitin sulfate; DS, dermatan sulfate; HS, heparan sulfate; KS, keratan sulfate; HA, hyaluronic acid; AM, amniotic membrane; FM, fetal membranes; IdoA, L-iduronic acid; GlcA, D-glucuronic acid; GalNAc, N-acetyl-D-galactosamine; FGF, fibroblast growth factor-2; PTN, pleiotrophin; MK, midkine; HGF, hepatocyte growth factor; P-ORN, poly-DL-ornithine; HPLC, high performance liquid chromatography; CSase, chondroitinase; NOP, neurite outgrowth-promoting; $\quad 2 \mathrm{AB}, \quad 2$-aminobenzamide; $\quad \Delta$ HexA, 4-deoxy-L-threo-hex-4-enepyranosyluronic acid; IU, international units;
\end{abstract}




\section{Introduction}

Glycosaminoglycans (GAGs) are involved in the regulation of various biological processes such as cell proliferation, differentiation, and migration, cell-cell recognition, extracellular matrix deposition, and tissue morphogenesis [1-4]. GAGs include chondroitin sulfate/dermatan sulfate (CS/DS), heparan sulfate (HS)/heparin, keratan sulfate (KS), and hyaluronic acid (HA). CS/DS chains are found ubiquitously in extracellular matrices and at cell surfaces. The sugar backbone of CS/DS consists of repeating disaccharide units: D-glucuronic acid (GlcA) $\beta / L$-iduronic acid (IdoA) $\alpha 1-3 N$-acetyl-D-galactosamine (GalNAc). During chain elongation in the biosynthesis of CS/DS, the disaccharide units are modified by specific sulfotransferases at C-2 of GlcA/IdoA, and C-4 and/or C-6 of GalNAc in various combinations, and display enormous structural diversity producing characteristic sulfation patterns critical for the binding to a variety of functional proteins [4]. This structural variability of GAGs is the basis for a wide range of their biological activities.

CS/DS plays crucial roles in various biological events such as the development of the central nervous system [4], wound healing [5, 6], and growth factor signaling $[3,7]$. Among these events, the involvement of CS/DS in the development of the central nervous system has attracted attention from a therapeutic point of view for the potential application of CS/DS to nerve regeneration. Several studies have shown that the sulfation pattern of brain CS/DS alters during development, characterized by an increase in 4-O-sulfation and a decrease in 6-O-sulfation in the whole brain [8], as well 
as a dramatic increase in the proportion of IdoA(2-O-sulfate)-GalNAc(4-O-sulfate) units in the cerebellum [9]. The proportion of oversulfated disaccharides and the presence of IdoA are crucial factors for the neurite outgrowth-promoting (NOP) activity of CS/DS [10-14]. CS/DS chains from embryonic pig brains contain a higher proportion of IdoA than those prepared from adult pig brains [12]. They promote the outgrowth of neurites of embryonic mouse hippocampal neurons in culture by capturing at least endogenous pleiotrophin (PTN) produced by non-neuronal cells (glial cells) and hepatocyte growth factor (HGF), and then presenting them to neuronal cells to promote neuritogenesis $[4,15,16]$.

The amniotic membrane (AM) is the innermost layer of fetal membranes (FMs) and possesses various biological activities. Since AM reduces ocular surface inflammation and scarring, and promotes rapid epithelialization, it has been applied to the treatment of several ocular disorders [17]. The proliferation of corneal epithelial cells was promoted when cultivated on human AM [18]. In addition, both mouse and human embryonic stem cells efficiently differentiate into neural precursors when cultured on human AM matrix components [19]. Although the mechanism underlying the biological activities of AM remains unclear, unique components seem to be involved. AM contains various extracellular matrix components such as type I collagen, laminin, fibronectin, HA, and proteoglycans including CS, HS, and KS chains [20]. Since GAGs have been implicated in various biological processes as described above, we hypothesized that GAGs in AM may play a major role in the biological activities of AM. 
Notably, CS/DS in AM may be involved in the induction of the differentiation of embryonic stem cells into neuronal precursors. Hence, in this study GAGs were isolated from porcine FMs containing amnions, and their structure and NOP activity were analyzed.

\section{Materials and methods}

Materials The following sugars and enzymes were purchased from Seikagaku Corp. (Tokyo, Japan): CS-E from squid cartilage, standard unsaturated disaccharides, chondroitinase (CSase) ABC (EC 4.2.2.4) from Proteus vulgaris, CSase AC-I (EC 4.2.2.5) from Flavobacterium heparinum, CSase AC-II (EC 4.2.2.5) from Arthrobactor aurescens, keratanase II (EC 3.2.1.) from Bacillus sp., and hyaluronidase (EC 4.2.2.1) from Streptomyces hyalurolyticus. CSase B (EC 4.2.2.19), and heparinases (Hepases) I (EC 4.2.2.7) and III (EC 4.2.2.8) from Flavobacterium heparinum were purchased from IBEX Technologies (Montreal, Canada). Porcine FMs were obtained from Hokudo Corp. (Hokkaido, Japan). Pregnant ddY mice were purchased from SLC Inc. (Shizuoka, Japan). Poly-DL-ornithine (P-ORN), anti-neurofilament and anti-HGF-antibodies, and serum immunoglobulins from mouse and goat, were purchased from Sigma. Actinase E was obtained from Kaken Pharmaceutical Co. (Tokyo, Japan). Anti-microtubule-associated protein 2 was purchased from Leico Technologies Inc. (St. Louis, MO). Recombinant human (rh)-PTN, rh-fibroblast growth factor-2 (FGF-2), and rh-HGF were purchased from Wako Corp. (Osaka, Japan). Anti-FGF-2 was from 
Upstate Biotechnology, Inc. (New York, NY). Anti-PTN and anti-midkine (MK) antibodies, and rh-MK were purchased from R\&D Systems (Minneapolis, MN). Accell Plus QMA anion-exchange cartridges were obtained from Waters Corp. Dialysis membranes were purchased form Spectrum laboratories Corp. (Rancho Dominguez, CA).

Extraction of the GAG-peptide fraction from porcine FMs Porcine FMs (350 g in wet weight) were cut into pieces with scissors, boiled in $650 \mathrm{ml}$ of water for $10 \mathrm{~min}$ to inactivate proteases and glycosidases, cooled, and digested with $3 \mathrm{~g}$ of actinase $\mathrm{E}$ in a total volume of $900 \mathrm{ml}$ of $0.1 \mathrm{M}$ borate-sodium buffer, $\mathrm{pH} 8.0$, containing $10 \mathrm{mM}$ calcium chloride as described previously [21]. The digestion was carried out at $60{ }^{\circ} \mathrm{C}$ for $96 \mathrm{~h}$, and an additional $1.5 \mathrm{~g}$ each of the enzyme was added after 24, 48, and $72 \mathrm{~h}$. Following incubation, the sample was precipitated with 5\% trichloroacetic acid and centrifuged. The supernatant was treated with diethylether to remove trichloroacetic acid. The aqueous phase was neutralized using $1 \mathrm{M}$ sodium carbonate, and treated with $80 \%$ ethanol containing $1 \%$ sodium acetate at $4{ }^{\circ} \mathrm{C}$ overnight. The resultant precipitate was reconstituted in $160 \mathrm{ml}$ of water, and subjected to anion-exchange chromatography on an Accell QMA Plus cartridge (Waters, Milford, MA), which was eluted stepwise with $50 \mathrm{mM}$ phosphate buffers, $\mathrm{pH} 6.0$, containing $0.15,1.0$, and $2.0 \mathrm{M} \mathrm{NaCl}$ [21]. Each eluted fraction was desalted using a Spectra/Por dialysis membrane (Spectrum laboratories Corp., Rancho Dominguez, CA). Uronic acid in each fraction was 
quantified by the carbazole method [22].

Purification of FM-CS/DS The fractions, which were eluted with buffers containing 1.0 and 2.0 M NaCl from an Accell QMA Plus cartridge, presumably contained GAG-peptides from FMs, and were digested with hyaluronidase from Streptomyces hyalurolyticus to eliminate $\mathrm{HA}$ as described previously [13]. The resultant HA fragments were removed by Centricon YM-10 (cut off molecular weight: 10,000) (Millipore, Bedford, MA). The 1.0 and 2.0 M NaCl-eluted fractions were then subjected to nitrous acid treatment to eliminate HS as reported previously [13], and the resultant HS fragments were removed by Centricon YM-10. The 1.0 and 2.0 M NaCl-eluted fractions were then digested with keratanase II in a $10 \mathrm{mM}$ sodium acetate buffer, $\mathrm{pH}$ 6.5 , at $37^{\circ} \mathrm{C}$ for $1 \mathrm{~h}$, and the resultant KS fragments were removed by Centricon YM-10. Finally, the purified fractions were treated with $1.0 \mathrm{M} \mathrm{NaBH}_{4} / 0.5 \mathrm{M} \mathrm{NaOH}$ at room temperature for $2 \mathrm{~h}$ to liberate peptides from GAG polysaccharides. The samples were acidified with acetic acid to decompose excess $\mathrm{NaBH}_{4}$, neutralized with $1 \mathrm{M}$ sodium carbonate, and passed through a Sep- $\mathrm{PakC}_{18}$ (Waters) cartridge to remove peptide fragments. The purified 1.0 and 2.0 M NaCl-eluted FM-CS/DS fractions were used for the structural and functional analyses.

Enzymatic digestion The GAG-peptide fractions from FMs, which were eluted using 1.0 and 2.0 $\mathrm{M} \mathrm{NaCl}$ from an Accell QMA Plus cartridge, were digested with a mixture of 
CSases ABC and AC-II, CSase B, a mixture of CSases AC-I and AC-II [13], or a mixture of Hepases I and III [23]. Unsaturated di- and oligosaccharides produced were derivatized with 2-aminobenzamide (2AB), and then analyzed by anion-exchange HPLC on an amine-bound silica PA-03 column (YMC Co., Kyoto, Japan) [24]. The GAG-peptide fractions from FMs were also digested with keratanase II as described above. The digests were derivatized with $2 \mathrm{AB}$, and then analyzed by anion-exchange HPLC on an amine-bound silica PA-03 column [24].

Gel-filtration chromatography of the FM-CS/DS preparations The FM-CS/DS preparations $(20 \mu \mathrm{g}$ each as CS/DS) were subjected to gel-filtration chromatography on a column of Sephacryl S-400 HR16/60 (GE healthcare, Uppsala, Sweden) using 0.2 $\mathrm{M}$ ammounium acetate as an effluent with a flow rate of $0.4 \mathrm{ml} / \mathrm{min}$. Fractions were collected at $1 \mathrm{~min}$ intervals, lyophilized, and digested with CSase $\mathrm{ABC}$. The digests were derivatized with $2 \mathrm{AB}$, and then analyzed by HPLC on an amine-bound silica column $[24]$.

Analysis of the distribution of IdoA residues in the FM-CS/DS preparations The 1.0 and 2.0 M NaCl-eluted FM-CS/DS preparations ( $1 \mu \mathrm{g}$ each as GAG) were digested with CSase AC-I. Each digest was labeled with $2 \mathrm{AB}$, and analyzed by gel-filtration HPLC on a column (10 X $300 \mathrm{~mm})$ of Superdex ${ }^{\mathrm{TM}}$ Peptide HR10/330 (Amersham Biosciences, Uppsala, Sweden), which was eluted with $0.2 \mathrm{M}$ ammonium bicarbonate at a flow rate 
of $0.4 \mathrm{ml} / \mathrm{min}$. The percentage of galactosaminidic bonds cleaved by CSase AC-I was calculated based on the fluorescent intensity across peaks relative to the total amount of the disaccharides generated by digestion with CSase $\mathrm{ABC}$ [12]. The molar ratio of IdoA to GlcA (IdoA/GlcA) was calculated based on the peak area as follows: (the amount of total disaccharides released by $\mathrm{CSase} \mathrm{ABC}$ minus the amount of oligo- and disaccharides released by CSase AC-I)/(the amount of oligo- and disaccharides released by CSase AC-I).

Assays for NOP activity Cultures of hippocampal cells were established from E16 mice as previously described $[11,25]$. Briefly, $2 \mu \mathrm{g} /$ well of a FM-CS/DS preparation was coated onto coverslips precoated with P-ORN at $4{ }^{\circ} \mathrm{C}$ overnight. The hippocampal cells were freshly isolated from E16 mouse embryos, suspended in Eagle's minimum essential medium containing N2 supplements [26], seeded on coverslips at a density of 20,000 cells $/ \mathrm{cm}^{2}$, and allowed to grow in a humidified atmosphere for $24 \mathrm{~h}$ at $37{ }^{\circ} \mathrm{C}$ with $5 \% \mathrm{CO}_{2}$.

For the inhibition assay using antibodies, polyclonal anti-PTN $(10 \mu \mathrm{g} / \mathrm{ml})$, anti-MK $(10 \mu \mathrm{g} / \mathrm{ml})$, anti-FGF-2 $(10 \mu \mathrm{g} / \mathrm{ml})$, or anti-HGF $(10 \mu \mathrm{g} / \mathrm{ml})$ antibodies were added to the medium, $2 \mathrm{~h}$ after the seeding of the hippocampal cells. After incubation overnight, the cells were fixed using $4 \%(\mathrm{w} / \mathrm{v})$ paraformaldehyde for $1 \mathrm{~h}$ at room temperature, and the neurites were visualized by immunochemical staining using anti-microtubule associated protein 2 (Lieco Technomogies Inc., St. Louis, MO) and 
anti-neurofilament (Sigma) antibodies as described [11]. The antibodies were then detected using a Vectastain ABC kit (Vector Laboratories Inc., Burlingame, CA) with 3,3'-diaminobenzidine as a chromogen. NOP activity was evaluated by determining the length of the longest neurite and the number of primary neurites of 100 randomly selected neurons using FLVFS-LS software (Olympus, Tokyo, Japan).

Surface plasmon resonance analysis The interaction of various growth/neurotrophic factors with the FM-CS/DS preparations was examined using a BIAcore X system (BIAcore AB, Uppsala, Sweden) as reported [12]. For the kinetic analysis, various concentrations of each growth/neurotrophic factor were injected onto the surface of the FM-CS/DS-immobilized sensor chip in the running buffer, pH 7.4 (HBS-EP, BIAcore $\mathrm{AB}$ ), containing $10 \mathrm{mM}$ HEPES, $0.15 \mathrm{M} \mathrm{NaCl}, 3 \mathrm{mM}$ EDTA, and $0.005 \%$ (w/v) Tween 20 at a flow rate of $30 \mu \mathrm{l} / \mathrm{min}$ [12]. Before each injection, base-line stabilization was achieved by injecting $1.0 \mathrm{M} \mathrm{NaCl}$ for $2 \mathrm{~min}$. The kinetic parameters were evaluated with BIAevalution software 4.1 (BIAcore $A B$ ) using a 1:1 binding model with mass transfer, and the association and dissociation rate constants $\left(k_{a}\right.$ and $\left.k_{d}\right)$ as well as the dissociation equilibrium constants $\left(K_{d}\right)$ were determined.

\section{Results}

Characterization of GAGs in porcine FMs To characterize GAGs in porcine FMs, a GAG-peptide fraction was prepared by digestion with actinase E, followed by 
trichloroacetic acid treatment and ethanol precipitation. This GAG-peptide fraction was further fractionated by anion-exchange chromatography using an Accell ${ }^{\mathrm{TM}}$ Plus QMA cartridge, which was eluted stepwise with buffers containing $0.15,1.0$, and $2.0 \mathrm{M} \mathrm{NaCl}$. Only trace amounts of GAGs were detected in the fraction eluted with the buffer containing $0.15 \mathrm{M} \mathrm{NaCl}$. The fractions eluted with 1.0 and $2.0 \mathrm{M} \mathrm{NaCl}$ were digested with a mixture of CSases ABC and AC-II, a mixture of Hepases I and III, or keratanse II. The digests were labeled with a fluorophore $2 \mathrm{AB}$ and analyzed by anion-exchange HPLC, and the GAG disaccharide composition in each fraction was quantified based on the fluorescent intensity of the derivatives (Table 1).

Representative chromatograms for the CS/DS disaccharide analysis are shown in Fig. 1, and the data are summarized in Table 2. The FM-CS/DS chains in the $1.0 \mathrm{M}$ $\mathrm{NaCl}$-eluted fraction were low sulfated as evidenced by a significant proportion of nonsulfated disaccharide unit, $\triangle \mathrm{HexA}$-GalNAc (40.5\%), with a sulfate/disaccharide unit ratio of 0.6 , whereas the FM-CS/DS chains in the $2.0 \mathrm{M} \mathrm{NaCl}$-eluted fraction were

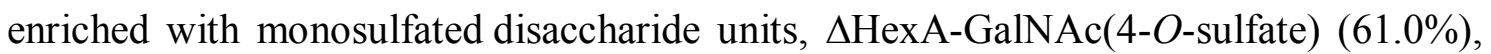
and $\Delta$ HexA-GalNAc(6-O-sulfate) (27.6\%), with a sulfate/disaccharide unit ratio of 0.9 (Table 2).

To examine whether the FM-GAG fractions contain DS, an anion-exchange HPLC analysis was carried out after digestion with a mixture of CSases AC-I and AC-II or CSase B alone, which specifically cleaves the $N$-acetylgalactosaminidic linkages flanked by GlcA or IdoA residues, respectively (Fig. 1 and Table 2). The GAG-peptide 
fractions were sensitive to the action of both types of enzymes, suggesting that the CS/DS chains have a hybrid structure.

Disaccharides derived from HA were also detected in the digest with a mixture of CSases $\mathrm{ABC}$ and $\mathrm{AC}$-II. The total amounts of HA disaccharides produced by digestion of the 1.0 and $2.0 \mathrm{M} \mathrm{NaCl}$-eluted GAG-peptide fractions with CSases were 1,085 and 104 pmol per mg of porcine FM (wet weight), respectively (Table 1). The GAG-peptide fractions were also subjected to a compositional analysis of HS disaccharides after digestion with a mixture of Hepases I and III. The total amounts of HS disaccharides produced were only 57 and 2 pmol per mg of porcine FM (wet weight), respectively. When the digest of the GAG-peptide fractions with keratanase II was analyzed by anion-exchange HPLC, KS disaccharides were detected only in the 2.0 $\mathrm{M} \mathrm{NaCl-eluted} \mathrm{GAG-peptide} \mathrm{fraction} \mathrm{and} \mathrm{the} \mathrm{amount} \mathrm{was} 11$ pmol per mg of porcine FM (wet weight).

The molecular size of CS/DS chains in the 1.0 and $2.0 \mathrm{M} \mathrm{NaCl-eluted}$ fractions was analyzed by gel-filtration chromatography. An aliquot of each eluate was subjected to digestion with $\mathrm{CS}$ ase $\mathrm{ABC}$ and the digests were labeled with $2 \mathrm{AB}$ and analyzed by anion-exchange HPLC. The amount of the $2 \mathrm{AB}$-derivatives of unsaturated CS/DS disaccharides was calculated based on fluorescent intensity. Using a calibration plot generated with data obtained with polysaccharides of known sizes (Fig. 2, inset), the average molecular size of the major CS/DS chains in the 1.0 and $2.0 \mathrm{M} \mathrm{NaCl}$-eluted fractions was estimated to be 301 and $249 \mathrm{kDa}$, respectively (Fig. 2). 
To further characterize the structural properties of the IdoA-containing FM-CS/DS preparations, the distribution of IdoA residues along the chains was compared between the 1.0 and $2.0 \mathrm{M}$ FM-CS/DS preparations. Each FM-CS/DS preparation was treated with CSase AC-I, and each digest was labeled with $2 \mathrm{AB}$ and analyzed by gel-filtration HPLC on a column of Superdex peptide. As shown in Fig. 3, fluorescent peaks derived from each digest were detected at the elution of positions corresponding to hexa- and tetrasaccharides, in addition to di-, mono-, and nonsulfated disaccharides. Considering the substrate specificity of CSase AC-I (27), the oligosaccharide resistant to CSase AC-I is most likely derived from the sequences containing IdoA residues. Upon digestion of the 1.0 M NaCl-eluted FM-CS/DS fraction, disaccharides were predominantly detected as shown in Fig. 3A. On the other hand, the digestion of the $2.0 \mathrm{M}$ NaCl-eluted FM-CS/DS fraction with CSase AC-I gave oligosaccharides ranging in size from disaccharides to polysaccharides (Fig. 3B). The oligosaccharides in the digest obtained with CSase AC-I are composed of contiguous IdoA-containing disaccharide units. The molar ratio of IdoA to GlcA plus IdoA was calculated based on the peak area, and in the 1.0 and $2.0 \mathrm{M} \mathrm{NaCl}$-eluted FM-CS/DS fractions was 0.23 and 0.62 , respectively. These results suggest that the proportion of the characteristic DS domain constituted of IdoA-containing disaccharide units is approximately 3 times higher in the $2.0 \mathrm{M}$ than $1.0 \mathrm{M} \mathrm{NaCl}$-eluted FM-CS/DS fraction.

NOP activity of the FM-CS/DS preparations To evaluate the biological activity of the 
CS/DS chains in FM, they were purified from the GAG-peptide fractions of porcine FM. HA, HS and KS were removed from the GAG-peptide fractions by digestion with hyaluronidase, treatment with nitrous acid, and digestion with keratanase II, respectively. The purified fractions were treated with $1.0 \mathrm{M} \mathrm{NaBH}_{4} / 0.5 \mathrm{M} \mathrm{NaOH}$ at room temperature for $2 \mathrm{~h}$ to liberate CS/DS chains from the core peptides, and the products were passed through a Sep-PakC $\mathrm{C}_{18}$ cartridge to remove peptide fragments.

The purified 1.0 and 2.0 M NaCl-eluted FM-CS/DS fractions were assessed for NOP activity. Mouse E16 hippocampal cells were cultured on plastic coverslips coated with P-ORN and then with the 1.0 or $2.0 \mathrm{M} \mathrm{NaCl-eluted} \mathrm{FM-CS/DS} \mathrm{fraction}$ (Figs. 4A and 4B) or CS-E (Fig. 4C) as a positive control. After incubation for $24 \mathrm{~h}$, the cells were fixed and immunostained with anti-microtuble-associated protein 2 and anti-neurofilament antibodies. In the negative control experiment, cells were cultured on coverslips coated with P-ORN alone (Fig. 4D). The mean length of the longest neurite and the number of primary neurites per cell of 100 randomly selected neurons cultured on various substrates were measured.

The mean length of the longest neurites was significantly greater for the neurons cultured on the FM-CS/DS preparations than for the cells cultured on coverslips coated with P-ORN alone (Fig. 5). The number of primary neurites was also larger for the neurons cultured on the FM-CS/DS preparations than those cultured on P-ORN alone (Fig. 5). Although only a single long neurite per neuron was usually observed for neurons cultured on P-ORN alone, neurons grown on the plates coated 
with the 1.0 and 2.0 M NaCl-eluted FM-CS/DS fractions projected 1.5 and 2.6 neurites per cell, respectively (Fig. 5B). The NOP activity level of the $2.0 \mathrm{M} \mathrm{NaCl-eluted}$ fraction was significantly higher than that of the $1.0 \mathrm{M} \mathrm{NaCl}$-eluted fraction. The NOP activity of the FM-CS/DS fractions was eliminated by digestion with CSase ABC (Fig. 5), suggesting the polymeric structure of CS/DS chains but not contaminants to indeed be required for the expression of the NOP activity.

Although both the FM-CS/DS preparations and CS-E displayed marked NOP effects (Fig. 4), the morphology of the neurons cultured on these substrates was different. The neurons cultured on the CS-E-coated substrate exhibited a round-shaped morphology (Fig. 4C), being consistent with previous observations [11]. On the other hand, the neurons cultured on the FM-CS/DS preparations extended multiple neurites and showed a flattened dendrite-like morphology (Figs. 4A and B) as observed for the neurons cultured on a CS-D-coated substrate [25].

CS/DS specifically interacts with PTN, MK, FGF-2, and HGF, which have neurotrophic activity and are widely expressed as autocrine and paracrine pleiotropic factors in distinct neuronal cell populations of the developing and adult brain, including hippocampal neuronal cells [28-30]. Hence, these endogenous heparin-binding growth factors may be involved in the mechanism of the NOP activity of FM-CS/DS. To verify this possibility, antibodies against HGF, PTN, MK, and FGF-2 were individually added and it was examined whether the NOP activity of FM-CS/DS chains toward hippocampal neurons was neutralized. The addition of anti-MK antibody markedly 
suppressed the NOP activity of 1.0 M NaCl-eluted FM-CS/DS chains to the background level of P-ORN, suggesting that the NOP activity of the $1.0 \mathrm{M} \mathrm{NaCl-eluted} \mathrm{FM-CS/DS}$ chains is expressed mainly through the signaling pathway of MK (Fig. 6A). On the other hand, the NOP activity of the $2.0 \mathrm{M} \mathrm{NaCl-eluted} \mathrm{FM-CS/DS} \mathrm{chains} \mathrm{was}$ suppressed by the antibodies against PTN and FGF-2 by 54 and 51\%, respectively, suggesting the involvement of the signaling pathways of PTN and FGF-2 in the expression of the NOP activity of the $2.0 \mathrm{M}$ NaCl-eluted FM-CS/DS chains.

Kinetic analysis of the binding of growth factors to FM-CS/DS The NOP activity of the FM-CS/DS chains was suppressed by the antibodies against PTN, MK, and FGF-2 (Fig. 6). Since CS/DS chains have been demonstrated to act as co-receptors for various growth factors [7], intractions with these heparin-binding growth factors may be involved in the promotion of neurite outgrowth by the FM-CS/DS chains. To examine this possibility, a kinetic analysis of the binding of these growth factors to immobilized FM-CS/DS chains was carried out using the BIAcore system. The FM-CS/DS preparations were biotinylated and immobilized on the streptavidin-precoated sensor chip. The heparin-binding growth factors (PTN, MK, FGF-2, and HGF) were injected at different concentrations individually onto the surface of the sensor chip. Overlaid sensorgrams are shown in Fig. 7. These sensorgrams were analyzed with BIAevaluation 4.1 software using the "1:1 binding model with mass tranfer" to calculate the kinetic parameters (Table 3). 
Although MK and FGF-2 bound to the $1.0 \mathrm{M} \mathrm{NaCl}$-eluted FM-CS/DS chains, PTN and HGF did not (data not shown). MK displayed quick binding and slow dissociation, giving a $K_{d}$ value of $27.1 \mathrm{nM}$ and signifying their strong affinity for the 1.0 M NaCl-eluted FM-CS/DS chains. On the other hand, all the growth factors tested bound to the $2.0 \mathrm{M} \mathrm{NaCl}$-eluted FM-CS/DS chains. PTN and MK showed high affinity, displaying $K_{d}$ values of 463.4 and $53.3 \mathrm{nM}$, respectively. The kinetic parameters for the binding of FGF-2 and HGF are not shown in Table 3, because good fitting models were not obtained for their sensorgrams (data not shown). However, the data on the binding of FGF-2 and HGF to the FM-CS/DS chains can be used qualitatively.

\section{Discussion}

In this study, to elucidate the biological activities of CS/DS chains in AM, the structure and bioactivity of FM-CS/DS chains were investigated. The disaccharide compositional analysis showed that the major components of the FM-CS/DS chains are GlcA-GalNAc, GlcA-GalNAc(4-O-sulfate), and GlcA-GalNAc(6-O-sulfate) disaccharide units, and that IdoA-containing disaccharide units are clustered in the polysaccharide chains. Because of the flexibility of its sugar ring, IdoA shows a conformational diversity [31, 32], which may provide IdoA-containing GAGs with a range of finely tuned affinities for different bioactive proteins [33]. DS, which mainly consists of IdoA-GalNAc(4-O-sulfate) disaccharide units, promotes the binding of FGF-7 to a FGF receptor and the cell proliferation of keratinocytes [6], and also acts as a co-receptor 
required for the formation of an active HGF signaling complex [34, 35]. The DS-like structure in Xenopus CS/DS was reported to be involved in its binding to FGF-1 [36]. The unusually flexible ring conformation of IdoA residues in FM-CS/DS may be involved in the biological activities of AM such as promotion of the proliferation of corneal epithelium [18] and regeneration of esophagus and skin [37, 38] mediated by growth factors' signaling.

Based on the analysis of the molecular sizes of the 1.0 and $2.0 \mathrm{M} \mathrm{NaCl}$-eluted FM-CS/DS preparations, the average molecular sizes of the 1.0 and $2.0 \mathrm{M} \mathrm{NaCl}$-eluted FM-CS/DS chains were estimated to be 301 and $249 \mathrm{kDa}$, respectively. These chains are linear polymers consisting of approximately 500 - 600 repeating disaccharide units and unusually larger than the CS/DS chains from other sources including embryonic pig brain $(40 \mathrm{kDa})$ [12], shark skin $(70 \mathrm{kDa})$ [13], shark liver (76 kDa) [16], and hagfish notochord (18 kDa) [14]. The markedly large CS chains (350 kDa) attach to type IX collagen in the chick embryo vitreous humor [39], which are presumed to distribute in a regular periodic arrangement along the collagen fibrils. AM has been utilized for the treatment of several corneal diseases such as corneal opacity and damaged corneas [18]. Since normal cornea abundantly contains collagen fibrils, the markedly large size of FM-CS/DS may contribute to the treatment of corneal diseases.

The NOP activity of the FM-CS/DS preparations was analyzed in this study to investigate potential applications to nerve regeneration. The $1.0 \mathrm{M} \mathrm{NaCl}$-eluted FM-CS/DS exhibited NOP activity with a dendrite-like morphology, and this activity 
was completely inhibited by the antibody against a neurotrophic factor MK, which showed a strong affinity for the $1.0 \mathrm{M} \mathrm{NaCl}$-eluted FM-CS/DS chains displaying a $K_{d}$ value of $53.3 \mathrm{nM}$. Several studies have demonstrated that endogenous growth/neurotrophic factors such as PTN, HGF, MK, and brain-derived neurotrophic factor are recruited by CS/DS chains and then presented to neuronal cells to promote neuritogenesis $[4,15,16,40]$. These findings suggest that endogenous MK appears to be recruited by the $1.0 \mathrm{M} \mathrm{NaCl}$-eluted FM-CS/DS chains and involved in the induction of the NOP activity. MK is a heparin-binding neurotrophic factor that promotes the growth, survival, and migration of various cells [41]. A cluster of GlcA-GalNAc(4,6-O-disulfate) units in CS-E from squid cartilage exhibits strong binding to $\mathrm{MK}\left(K_{d}=62\right)[42,43]$. However, the FM-CS/DS chains contain little GlcA-GalNAc(4,6-O-disulfate) unit $(0.03 \%)$, indicating that the functional domains in the 1.0 M NaCl-eluted FM-CS/DS chains for the MK-binding do not appear to contain GlcA-GalNAc(4S,6S) units, but rather a cluster of IdoA-containing disaccharides, which interacts with MK [12] and influences the biological activity of MK, may be involved [44].

The 2.0 M NaCl-eluted FM-CS/DS preparation also displayed marked NOP activity with a dendrite-like morphology, and its activity level was significantly higher than that of the $1.0 \mathrm{M}$ NaCl-eluted FM-CS/DS chains. A contribution of IdoA-containing disaccharide units to the neuritogenic activity of CS/DS chains has been demonstrated using CS/DS preparations derived from embryonic pig brain [12], 
shark skin [13], and hagfish notochord [14]. Consecutive IdoA residues appear to be essential for the NOP activity of the FM-CS/DS chains, being consistent with the observation that the $2.0 \mathrm{M} \mathrm{NaCl}$-eluted FM-CS/DS chains contain a higher proportion of IdoA-containing disaccharide units than the 1.0 M NaCl-eluted FM-CS/DS chains. The NOP activity of the 2.0 M NaCl-eluted FM-CS/DS chains was diminished by the antibodies against PTN and FGF-2 by 54 and 51\%, respectively, suggesting the involvement of the signaling pathways of PTN and FGF-2 in the expression of the NOP activity of the $2.0 \mathrm{M} \mathrm{NaCl}$-eluted FM-CS/DS chains. However, the NOP activity of the 2.0 M NaCl-eluted FM-CS/DS chains was not completely eliminated by the addition of the mixture of these antibodies (data not shown). Recently, Mikami et al. reported that CS-E can act as an effective ligand for the neuronal cell surface receptor contactin-1 that stimulates the intracellular signaling pathway involved in neuritogenesis [45]. Cell adhesion molecules such as contactin-1 may be involved in the induction of the NOP activity of the $2.0 \mathrm{M} \mathrm{NaCl-eluted} \mathrm{FM-CS/DS} \mathrm{chains} \mathrm{to} \mathrm{some} \mathrm{extent.}$

The matrix components of human AM can convert embryonic stem cells into neural tissues selectively [19]. Since CS/DS is a major component of the extracellular matrix, the NOP activity of the FM-CS/DS chains demonstrated in this study may be involved in the neurogenerative activity of AM. AM has also been applied to several ocular disorders, because it can promote epithelialization, inhibit fibrosis, and reduce ocular surface inflammation [17]. In addition, it has antimicrobial and antiviral properties and high hydraulic conductivity [17]. Basement membrane components in 
AM can improve the morphogenesis of epidermis and basement membranes in constructing a human living skin-equivalent [38]. The in situ tissue-engineered esophagus, which is prepared by cultivating oral keratinocytes and fibroblasts on human AM, can successfully replace the intrathoracic esophagus [37]. Since CS/DS is involved in biological events such as cell proliferation [3, 6], viral infection [46], inflammation [47], anti-coagulation [33], and wound healing [5, 6, 48], it may contribute to some of the biological activities of AM described above. The information obtained in this study facilitates deeper understanding of the biological activities of AM, and suggests potential applications of FM-CS/DS chains.

\section{Acknowledgements}

This work was supported by Grant-in-aid for Scientific Research (B) 20390019 (to K.

S.) from MEXT (Ministry of Education, Culture, Sports, Science and Technology, Japan), and Sapporo Biocluster "Bio-S", the Knowledge Cluster Initiative of the MEXT. 


\section{Footnotes}

1. Affiliation of the authors

Taishi Hashiguchi ${ }^{1}$, Shuji Mizumoto ${ }^{1}$, Shuhei Yamada ${ }^{1, *}$, and Kazuyuki Sugahara ${ }^{1, *}$

(1) Laboratory of Proteoglycan Signaling and Therapeutics, Graduate School of Life

Science, Hokkaido University, Frontier Research Center for Post-Genomic Science and Technology, Nishi 11-choume, Kita 21-jo, Kita-ku, Sapporo, Hokkaido 001-0021, Japan *E-mail: tjohej@sci.hokudai.ac.jp and k-sugar@sci.hokudai.ac.jp 


\section{References}

1. Sasisekharan, R., Raman, R., and Prabhakar, V.: Glycomics approach to structure-function relationships of glycosaminoglycans. Annu. Rev. Biomed. Eng. 8, 181-231 (2006)

2. Sugahara, K., Mikami, T., Uyama, T., Mizuguchi, S., Nomura, K., and Kitagawa, H.: Recent advances in the structural biology of chondroitin sulfate and dermatan sulfate. Curr. Opin. Struct. Biol. 13, 612-620 (2003)

3. Nandini, C. D., and Sugahara, K.: Role of the sulfation pattern of chondroitin sulfate in its biological activities and in the binding of growth factors. Adv. Pharmacol. 53, 253-279 (2006)

4. Sugahara, K., and Mikami T.: Chondroitin sulfate/dermatan sulfate in the central nervous system. Curr. Opin. Struct. Biol. 17, 536-545 (2007)

5. Penc, S. F., Pomahac, B., Winkler, T., Dorschner, R. A., Eriksson, E., Herndon, M., and Gallo, R. L.: Dermatan sulfate released after injury is a potent promoter of fibroblast growth factor-2 function. J. Biol. Chem. 273, 28116-28121 (1998)

6. Trowbridge, J. M., Rudisill, J. A., Ron, D., and Gallo, R. L.: Dermatan sulfate binds and potentiates activity of keratinocyte growth factor (FGF-7). J. Biol. Chem. 277, 42815-42820 (2002)

7. Yamada, S., and Sugahara, K.: Potential therapeutic application of chondroitin sulfate/dermatan sulfate. Curr. Drug Discov. Technol. 5, 289-301 (2008)

8. Kitagawa, H., Tsutsumi, K., Tone, Y., and Sugahara, K.: Developmental regulation 
of the sulfation profile of chondroitin sulfate chains in the chicken embryo brain. J. Biol. Chem. 272, 31377-31381 (1997)

9. Mitsunaga, C., Mikami, T., Mizumoto, S., Fukuda, J., and Sugahara, K.: Chondroitin sulfate/dermatan sulfate hybrid chains in the development of cerebellum. J. Biol. Chem. 281, 18942-18952 (2006)

10. Nadanaka, S., Clment, A. M., Masayama, K., Faissner, A., and Sugahara, K.: Characteristic hexasaccharide sequences in octasaccharides derived from shark cartilage chondroitin sulfate D with a neurite outgrowth promoting activity. J. Biol. Chem. 273, 3296-3307 (1998)

11. Hikino, M., Mikami, T., Faissner, A., Vilela-Silva, A. C., Pava õ, M. S. G., and Sugahara, K.: Oversulfated dermatan sulfate exhibits neurite outgrowth-promoting activity toward embryonic mouse hippocampal neurons. J. Biol. Chem. 278, 43744-43754 (2003)

12. Bao, X., Nishimura, S., Mikami, T., Yamada, S., Itoh, N., and Sugahara, K.: Chondroitin sulfate/dermatan sulfate hybrid chains from embryonic pig brain, which contain a higher proportion of L-iduronic acid than those from adult pig brain, exhibit neuritogenic and growth factor binding activities. J. Biol. Chem. 279, 9765-9776 (2004)

13. Nandini, C. D., Itoh, N., and Sugahara, K.: Novel 70-kDa chondroitin sulfate/dermatan sulfate hybrid chains with a unique heterogenous sulfation pattern from shark skin, which exhibit neuritogenic activity and binding activities for 
growth factors and neurotrophic factors. J. Biol. Chem. 280, 4058-4069 (2005)

14. Nandini, C. D., Mikami, T., Ohta, M., Itoh N., Akiyama-Nambu, F., and Sugahara, K.: Structural and functional characterization of oversulfated chondroitin sulfate/dermatan sulfate hybrid chains from the notochord of hagfish. J. Biol. Chem. 279, 50799-50809 (2004)

15. Bao, X., Mikami, T., Yamada, S., Faissner, A., Muramatsu, T., and Sugahara, K.: Heparin-binding growth factor, pleiotrophin, mediates neuritogenic activity of embryonic pig brain-derived chondroitin sulfate/dermatan sulfate hybrid chains. J. Biol. Chem. 280, 9180-9191 (2005)

16. Li, F., Shetty K. A., and Sugahara, K.: Neuritogenic activity of chondroitin sulfate/dermatan sulfate hybrid chains of embryonic pig brain and their mimicry from shark liver. J. Biol. Chem. 282, 2956-2966 (2007)

17. Fernandes, M., Sridhar, S. M., Sangwan, S. V., and Rao, N. G.: Amniotic membrane transplantation for ocular surface reconstruction. Cornea 24, 643-653 (2005)

18. Koizumi, N., Fullwood, J. N., Bairaktaris, G., Inatomi, T., Kinoshita, S., and Quantock, J. A.: Cultivation of corneal epithelial cells on intact and denuded human amniotic membrane. Invest. Ophthalmol. Vis. Sci. 41, 2506-2513 (2000)

19. Ueno, M., Matsumura, M., Watanabe, K., Nakamura, T., Osakada, F., Takahashi, M., Kawasaki, H., Kinoshita S., and Sasai, Y. Neural conversion of ES cells by an inductive activity on human amniotic membrane matrix. Proc. Natl. Acad. Sci. U. 
20. Cooper, L. J., Kinoshita, S., German, M., Koizumi, N., Nakamura, T., and Fullwood, N. J.: An investigation into the composition of amniotic membrane used for ocular surface reconstruction. Cornea 24, $722-729$ (2005)

21. Yamada, S., Okada, Y., Ueno, M., Iwata, S., Deepa, S. S., Nishimura, S., Fujita, M., Irma, V. D., Hirabayashi, Y., and Sugahara, K.: Determination of the glycosaminoglycan-protein linkage region oligosaccharide structures of proteoglycans from Drosophila melanogaster and Caenorhabditis elegans. J. Biol. Chem. 277, 31877-31886 (2002)

22. Bitter, T., and Muir, H.: A modified uronic acid carbazole reaction. Anal. Biochem. 4, 330-334 (1962)

23. Ueno, M., Yamada, S., Zako, M., Bernfield, M., and Sugahara, K.: Structural characterization of heparan sulfate and chondroitin sulfate of syndecan-1 purified from normal murine mammary gland epithelial cells. J. Biol. Chem. 276, 29134-29140 (2001)

24. Kinoshita, A., and Sugahara, K.: Microanalysis of glycosaminoglycan-derived oligosaccharides labeled with a fluorophore 2-aminobenzamide by high-performance liquid chromatography: application to disaccharide composition analysis and exosequencing of oligosaccharides. Anal. Biochem. 269, 367-378 (1999)

25. Clement, M. A., Nadanaka, S., Masayama, M., Mandl, C., Sugahara K., and 
Faissner, A.: The DSD-1 carbohydrate epitope depends on sulfation, correlates with chondroitin sulfate D motifs, and is sufficient to promote neurite outgrowth. J. Biol. Chem. 273, 28444-35811 (1998)

26. Bottenstein, J. E., and Sato, G. H.: Growth of a rat neuroblastoma cell line in serum-free supplemented medium. Proc. Natl. Acad. Sci. U. S. A. 76, 514-517 (1979)

27. Saito, H., Yamagata, T., and Suzuki, S.: Enzymatic methods for the determination of small quantities of isomeric chondroitin sulfates. J. Biol. Chem. 243, 1536-1542 (1968)

28. Jung, W., Castren, E., Odenthal, M., Vande, W. G. F., Ishii, T., Dienes, H. P., Lindholm, D., and Schirmacher, P.: Expression and functional interaction of hepatocyte growth factor-scatter factor and its receptor c-met in mammalian brain. J. Cell Biol. 126, 485-494 (1994)

29. Böhlen, P., and Kovesdi, I.: HBNF and MK, members of a novel gene family of heparin-binding proteins with potential roles in embryogenesis and brain function. Prog. Growth Factor Res. 3, 143-157 (1991)

30. Haynes, L. W.: Developmental and regional expression of basic fibroblast growth factor mRNA in the rat central nervous system. Mol. Neurobiol. 2, 263-289 (1988)

31. Casu, B., Petitous, M., Provasoli, M., and Sinay, P.: Conformational flexibility: a new concept for explaining binding and biological properties of iduronic acid-containing glycosaminoglycans. Trends Biochem. Sci. 13, 747-751 (1988) 
32. Mulloy, B., and Forster, M.: Conformation and dynamics of heparin and heparan sulfate. Glycobiology 10, 1147-1156 (2000)

33. Trowbridge, J. M., and Gallo, R. L.: Dermatan sulfate: new functions from an old glycosaminoglycan. Glycobiology 12, 1147-1156 (2002)

34. Bechard, D., Gentina, T., Delehedde, M., Scherpereel, A., Lyon, M., Aumercier, M., Vazeux, R., Richet, C., Degand, P., Jude, B., Jani, A., Fernig, D. G., Tonnel, A. B., and Lassalle, P.: Endocan is a novel chondroitin sulfate/dermatan sulfate proteoglycan that promotes hepatocyte growth factor/scatter factor mitogenic activity. J. Biol. Chem. 276, 48341-48349 (2001)

35. Catlow, K. R., Deakin, J. A., Wei, Z., Delehedde, M., Fernig, D. G., Gherardi, E., Gallagher, J. T., Pavão M. S., and Lyon M.: Interactions of hepatocyte growth factor/scatter factor with various glycosaminoglycans reveal an important interplay between the presence of iduronate and sulfate density. J. Biol. Chem. 283, $5235-5248(2008)$

36. Yamada, S., Onishi, M., Fujinawa, R., Tadokoro, Y., Okabayashi, K., Asashima, M., and Sugahara, K.: Structural and Functional Changes of Sulfated Glycosaminoglycans in Xenopus laevis during Embryogenesis . Glycobiology in press (2009)

37. Nakase, Y., Nakamura, T., Kin, S., Nakashima, S., Yoshikawa, T., Kuriu, Y., Sakakura, C., Yamagishi, H., Hamuro, J., Ikada, Y., Otsuji, E., and Hagiwara, A.: Intrathoracic esophageal replacement by in situ tissue-engineered esophagus. J. 
Thorac. Cardiovasc. Surg. 136, 850-859 (2008)

38. Yang, L., Shirakata, Y., Shudou, M., Dai, X., Tokumaru, S., Hirakawa, S., Sayama, K., Hamuro, J., and Hashimoto, K.: New skin-equivalent model from de-epithelialized amnion membrane. Cell Tissue Res. 326, 69-77 (2006)

39. Yada, T., Suzuki, S., Kobayashi, K., Kobayashi, M., Hoshino, T., Horie, K., and Kimata, K.: Occurrence in chick embryo vitreous humor of a type IX collagen proteoglycan with an extraordinarily large chondroitin sulfate chain and short al polypeptide. J. Biol. Chem. 265, 6992-6999 (1990)

40. Sotogaku, N., Tully, S. E., Gama, C. I., Higashi, H., Tanaka, M., Heieh-Wilson, L. C., and Nishi, A.: Activation of phospholipase C pathways by a synthetic chondroitin sulfate-E tetrasaccharide promotes neurite outgrowth of dopaminergic neurons. J. Neurochem. 103, 749-760 (2007)

41. Muramatsu, T. Midkine and pleiotrophin: two related proteins involved in development, survival, inflammation and tumorigenesis. J. Biochem. 132, 359-371 (2002)

42. Deepa, S. S., Umehara, Y., Higashiyama, S., Itoh, N., and Sugahara, K.: Specific molecular interactions of oversulfated chondroitin sulfate $E$ with various heparin-binding growth factors. J. Biol. Chem. 277, 43707-43716 (2002)

43. Zou, P., Zou, K., Muramatsu, H., Ichihara-Tanaka, K., Habuchi, O., Ohtake, S., Ikematsu, S., Sakuma, S., and Muramatsu, T.: Glycosaminoglycan structures required for strong binding to midkine, a heparin-binding growth factor. 
Glycobiology 13, 35-42 (2003)

44. Hayashi, K., Kadomatsu, K., and Muramatsu, T.: Requirement of chondroitin sulfate/dermatan sulfate recognition in midkine-dependent migration of macrophages. Glycoconj. J. 18, 401-406 (2001)

45. Mikami, T., Yasunaga, D., and Kitagawa, H.: Contactin-1 is a functional receptor for neuroregulatory chondroitin sulfate-E. J. Biol. Chem. in press (2009)

46. Uyama, T., Ishida, M., Izumikawa, T., Trybala, E., Tufaro, F., Bergström, T., Sugahara, K., and Kitagawa, H.: Chondroitin 4-O-sulfotransferase-1 regulates E disaccharide expression of chondroitin sulfate required for herpes simplex virus infectivity. J. Biol. Chem. 281, 38668-38674 (2006)

47. Taylor, K. R., and Gallo, R. L.: Glycosaminoglycans and their proteoglycans:host-associated molecular patterns for initiation and modulation of inflammation. FASEB J. 20, 9-22 (2006)

48. Zou, H. X., Jiang, Z. Y., Zhang, R. G., Jin, M. H., Hieu, M. T. N., and Ouyang, W. H.: Specific interactions between human fibroblasts and particular chondroitin sulfate molecules for wound healing. Acta Biomater. in press (2009) 


\section{Figure legends}

Fig. 1 Anion-exchange HPLC of CSase digests of the 2.0 M NaCl-eluted FM-CS/DS fraction. The $2.0 \mathrm{M} \mathrm{NaCl-eluted} \mathrm{FM-CS/DS} \mathrm{fraction} \mathrm{was} \mathrm{digested} \mathrm{with} \mathrm{a} \mathrm{mixture} \mathrm{of}$ CSases ABC and AC-II (A), a mixture of CSases AC-I and AC-II (B), or CSase B (C). Each digest was labeled with $2 \mathrm{AB}$, and analyzed by anion-exchange HPLC on an amine-bound silica PA-03 column using a linear $\mathrm{NaH}_{2} \mathrm{PO}_{4}$ gradient (indicated by the dashed line). The elution positions of authentic $2 \mathrm{AB}-$ labeled unsaturated diasaccharides are indicated by bars. 1, $\Delta$ HexA-GlcNAc; 2, $\Delta$ HexA-GalNAc; 3, $\Delta$ HexA -GalNAc(6-O-sulfate); 4, $\Delta$ HexA-GalNAc(4-O-sulfate); 5, $\Delta \mathrm{HexA}(2-O$-sulfate) -GalNAc(6-O-sulfate); $\quad 6, \quad \Delta \mathrm{HexA}(2-O$-sulfate)-GalNAc(4-O-sulfate); $\quad$, $\quad \Delta$ HexA -GalNAc(4,6-O-disulfate). The peaks marked by asterisks are derived from the 2AB-labeling reagents. The insets in $(\mathrm{A})$ and $(\mathrm{C})$ show enlarged views (100-fold and 20-fold, respectively).

Fig. 2 Determination of the molecular size of the FM-CS/DS chains by gel-filtration chromatography. The FM-CS/DS preparations eluted with buffers containing 1.0 or 2.0 $\mathrm{M} \mathrm{NaCl}(20 \mu \mathrm{g}$ of each as GAG) were fractionated on a column of Sephacryl S-400 using $0.2 \mathrm{M}$ ammonium acetate as an effluent with a flow rate of $1 \mathrm{ml} / \mathrm{min}$. Fractions were collected at 2 min intervals. Each fraction was subjected to digestion with CSase $\mathrm{ABC}$ and labeled with $2 \mathrm{AB}$. The $2 \mathrm{AB}$-labeled digest was analyzed by anion-exchange HPLC on an amine-bound silica PA-03 column. The amount of the 2AB-derivatives of 
unsaturated CS/DS disaccharides was calculated based on fluorescent intensity. The inset shows a calibration curve giving a linear relationship between $\log \mathrm{M}_{\mathrm{r}}$ and elution time, which was prepared using size-defined commercial polysaccharides; dextran (average $\left.\mathrm{M}_{\mathrm{r}}: 200,000,70,000,18,100\right) . V_{0}$ and $V_{t}$ represent the void volume and the total volume, respectively.

Fig. 3 Analysis of the distribution of IdoA residues in the FM-CS/DS preparations.

The 1.0 M (A) and 2.0 M NaCl-eluted (B) FM-CS/DS preparations (1 $\mu \mathrm{g}$ of each as GAG) were digested with CSase AC-I, and the digests were labeled with $2 \mathrm{AB}$ and analyzed by gel-filtration HPLC on a Superdex peptide column. $\mathrm{V}_{0}$, void volume; $\mathrm{V}_{\mathrm{t}}$, total volume. The elution positions of authentic unsaturated CS oligosaccharides labeled with $2 \mathrm{AB}$ are indicated by bars: 1 , hexasaccharides; 2 , tetrasaccharides; 3 , trisulfated disaccharide; 4, disulfated disaccharides; 5 , monosulfated disaccharides; 6 , nonsulfated disaccharide.

Fig. 4 Morphology of E16 mouse hippocampal neurons cultured on coverslips coated with the FM-CS/DS preparations. E16 mouse hippocampal cells $\left(20,000\right.$ cells $\left./ \mathrm{cm}^{2}\right)$ were cultured on plastic coverslips coated with P-ORN and then with the $1.0 \mathrm{M}(\mathrm{A})$ or 2.0 M (B) NaCl-eluted FM-CS/DS preparation, or CS-E (C) as a positive control (2 $\mu \mathrm{g}$ of each as GAG). The cells were fixed and stained with anti-microtubule-associated protein 2 and anti-neurofilament antibodies. In the control experiments, cells were 
cultured on coverslips coated with P-ORN alone (D). Scale bar, $50 \mu \mathrm{m}$.

Fig. 5 NOP activity of the FM-CS/DS preparations. E16 mouse hippocampal cells $\left(20,000\right.$ cells $\left./ \mathrm{cm}^{2}\right)$ were cultured for $24 \mathrm{~h}$ on plastic coverslips coated with P-ORN and then with the 1.0 or $2.0 \mathrm{M} \mathrm{NaCl}$-eluted FM-CS/DS preparation, or with their CSase $\mathrm{ABC}$ digests $(2 \mu \mathrm{g}$ of each as GAG). The cells were fixed and stained with anti-microtubule-associated protein 2 and anti-neurofilament antibodies. In the control experiments, cells were cultured on coverslips coated with P-ORN alone. The mean length of the longest neurite per cell (A) and the number of primary neurites per cell (B) of 100 randomly selected neurons cultured on various substrates were measured (see "Materials and methods"). The statistical analysis was performed using Mann-Whitney's $U$ test (n.s., not significant; *,$p<0.01$; $^{*}, p<0.001$ ).

Fig. 6 Effects of antibodies against growth factors and neurotrophic factors on the NOP activity of the FM-CS/DS preparations. E16 mouse hippocampal cells $(20,000$ cells $/ \mathrm{cm}^{2}$ ) were cultured on plastic coverslips coated with P-ORN and then with the 1.0 or 2.0 M NaCl-eluted FM-CS/DS preparation ( $2 \mu \mathrm{g}$ of each as GAG). Two hours after seeding, anti-PTN (10 $\mu \mathrm{g} / \mathrm{ml})$, anti-MK $(10 \mu \mathrm{g} / \mathrm{ml})$, anti-FGF-2 $(10 \mu \mathrm{g} / \mathrm{ml})$, or anti-HGF $(10 \mu \mathrm{g} / \mathrm{ml})$ antibodies were added to the culture, and incubated for $24 \mathrm{~h}$. The mean length of the longest neurite was evaluated as described in the legend to Fig. 5. The same amount of an IgG from the corresponding animal serum (mouse or goat) was used 
as a negative control. Values represent the means \pm S.D. obtained from three independent experiments.

Fig. 7 Binding of various growth factors to immobilized FM-CS/DS chains. Various concentrations of MK (A, D), FGF-2 (B, E), PTN (C), or HGF (F) were injected onto the surface of a sensor chip immobilized with $1.0 \mathrm{M}(\mathrm{A}, \mathrm{B})$ or $2.0 \mathrm{M}$ NaCl-eluted FM-CS/DS chains (C-F). Sensograms obtained with various concentrations of each growth/neurotrophic factor were overlaid using BIAevaluation software (version 4. 1). 
Figure1

Click here to download high resolution image

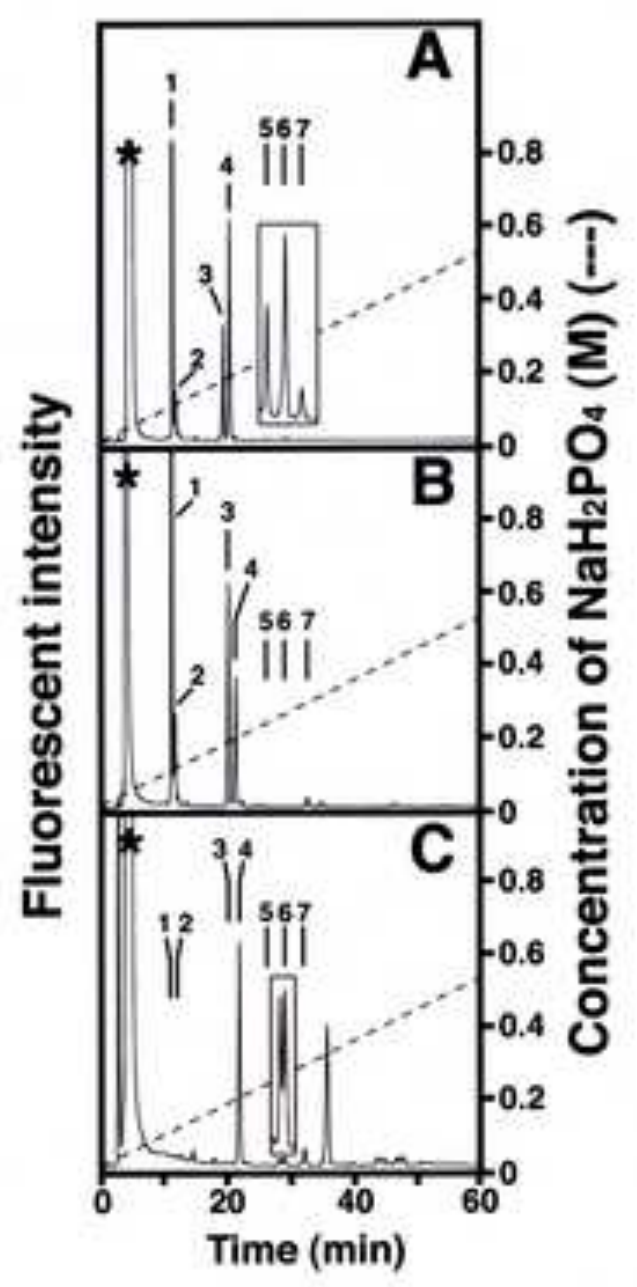


Figure2

Click here to download high resolution image

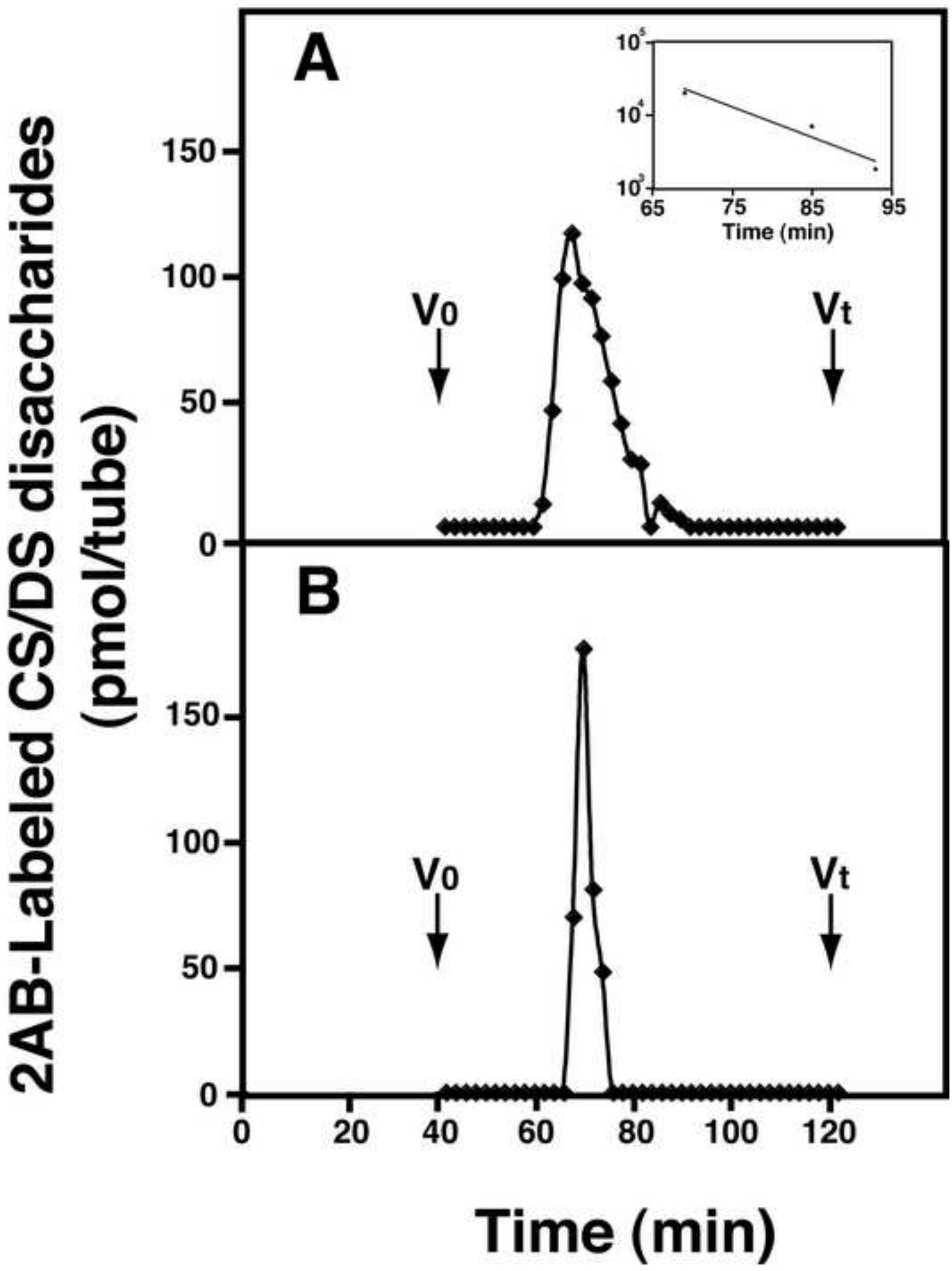


Click here to download high resolution image

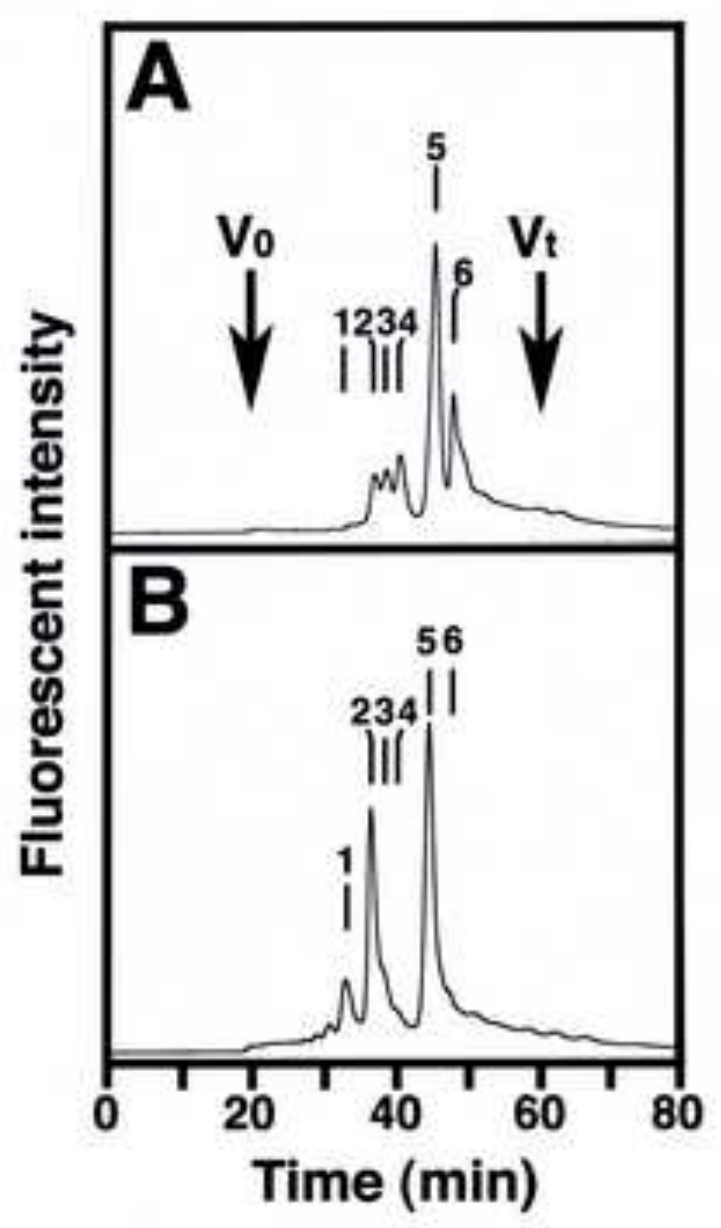


Click here to download high resolution image
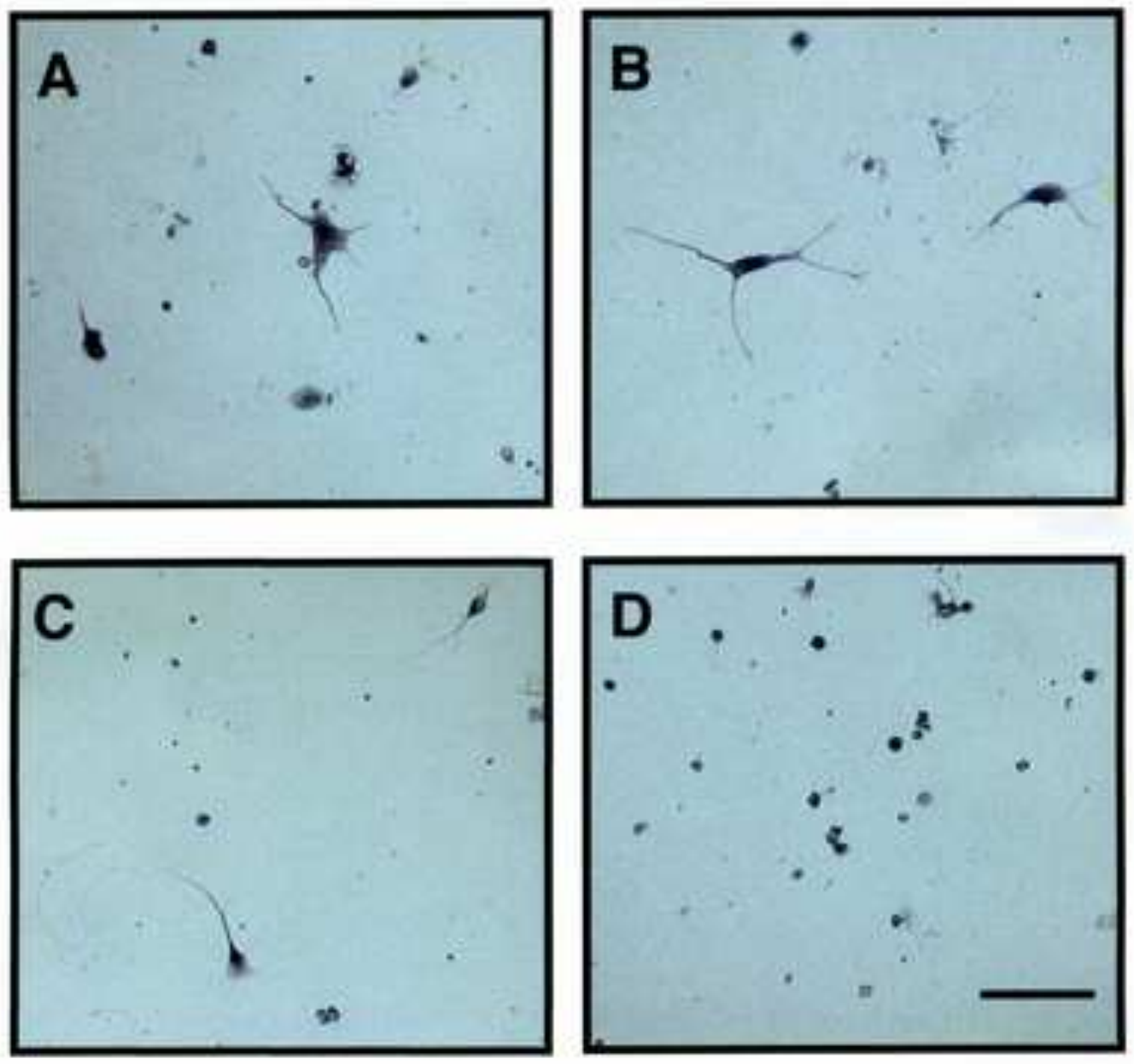

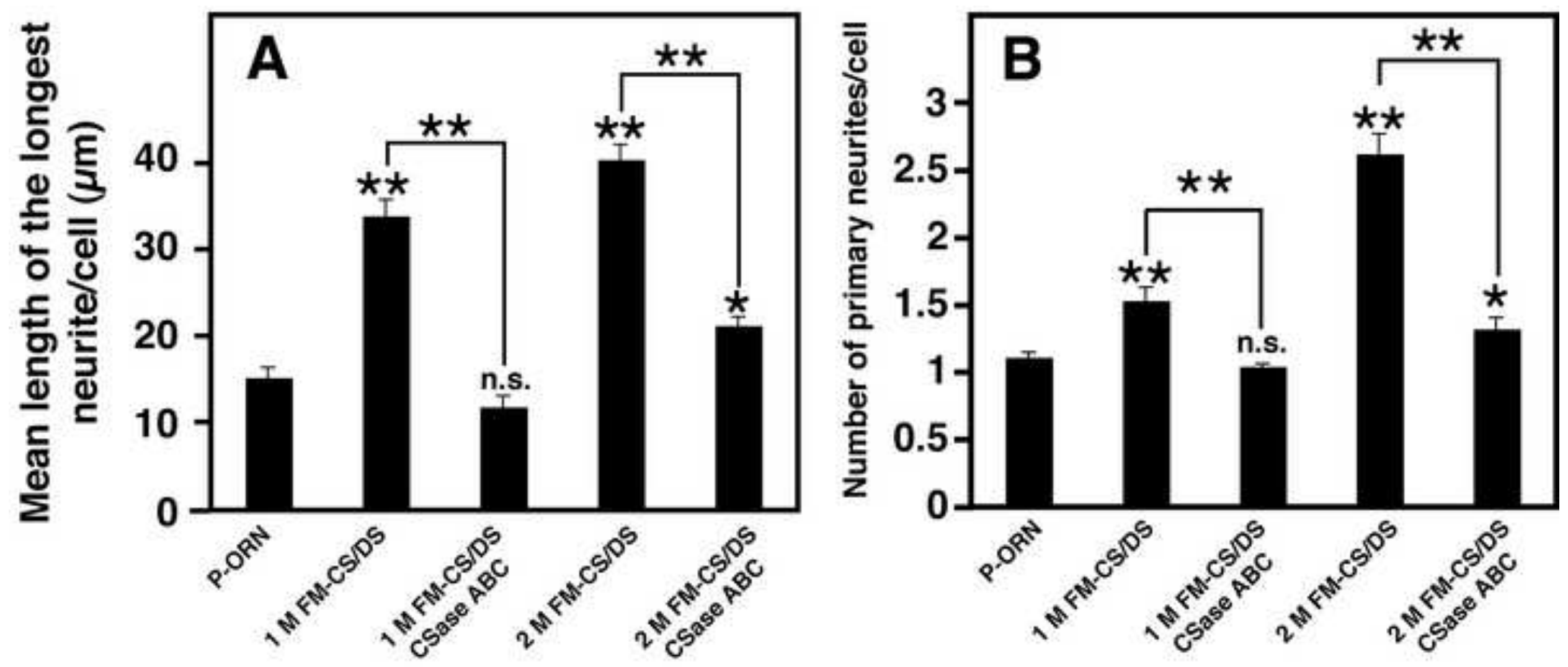


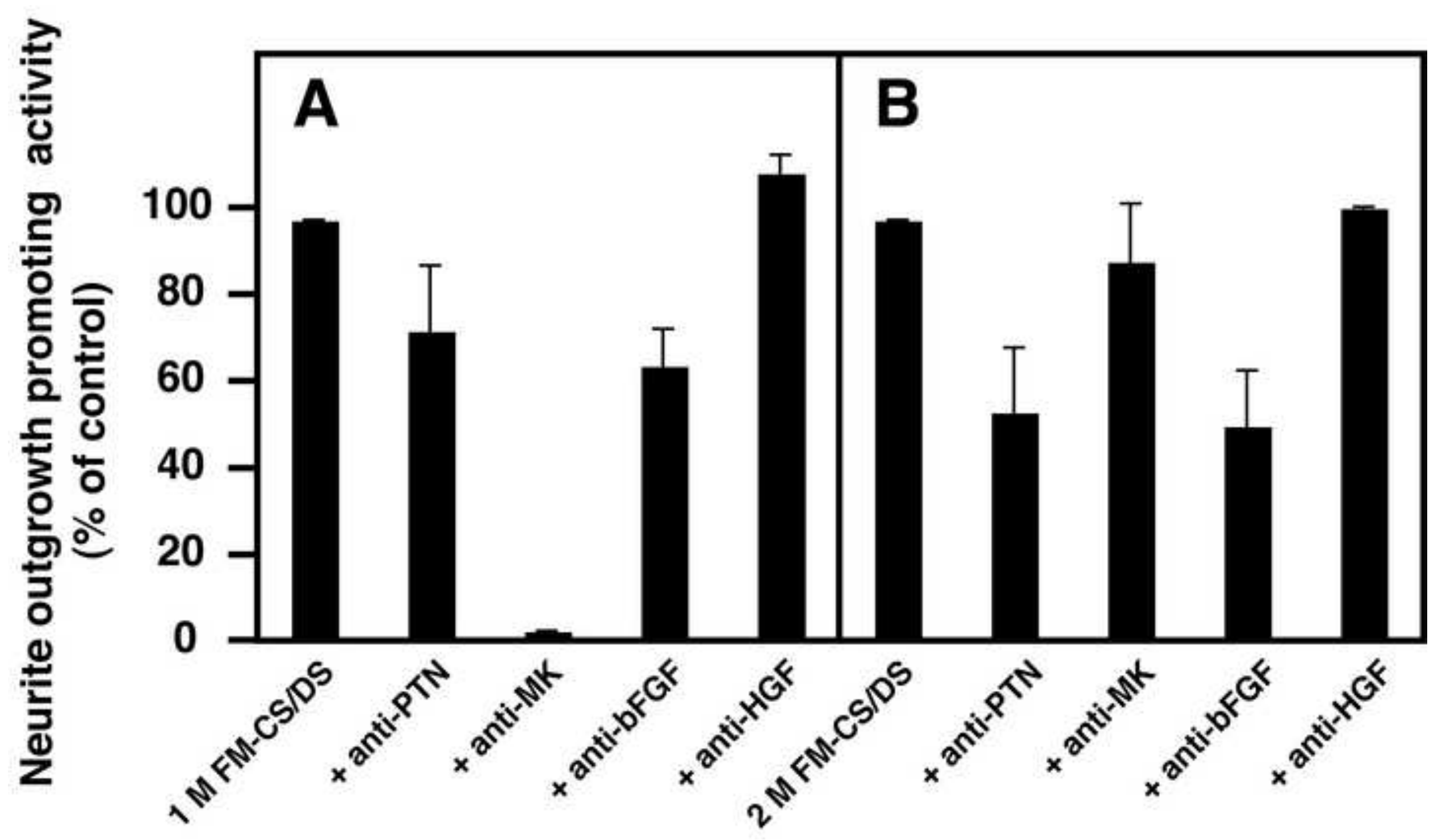




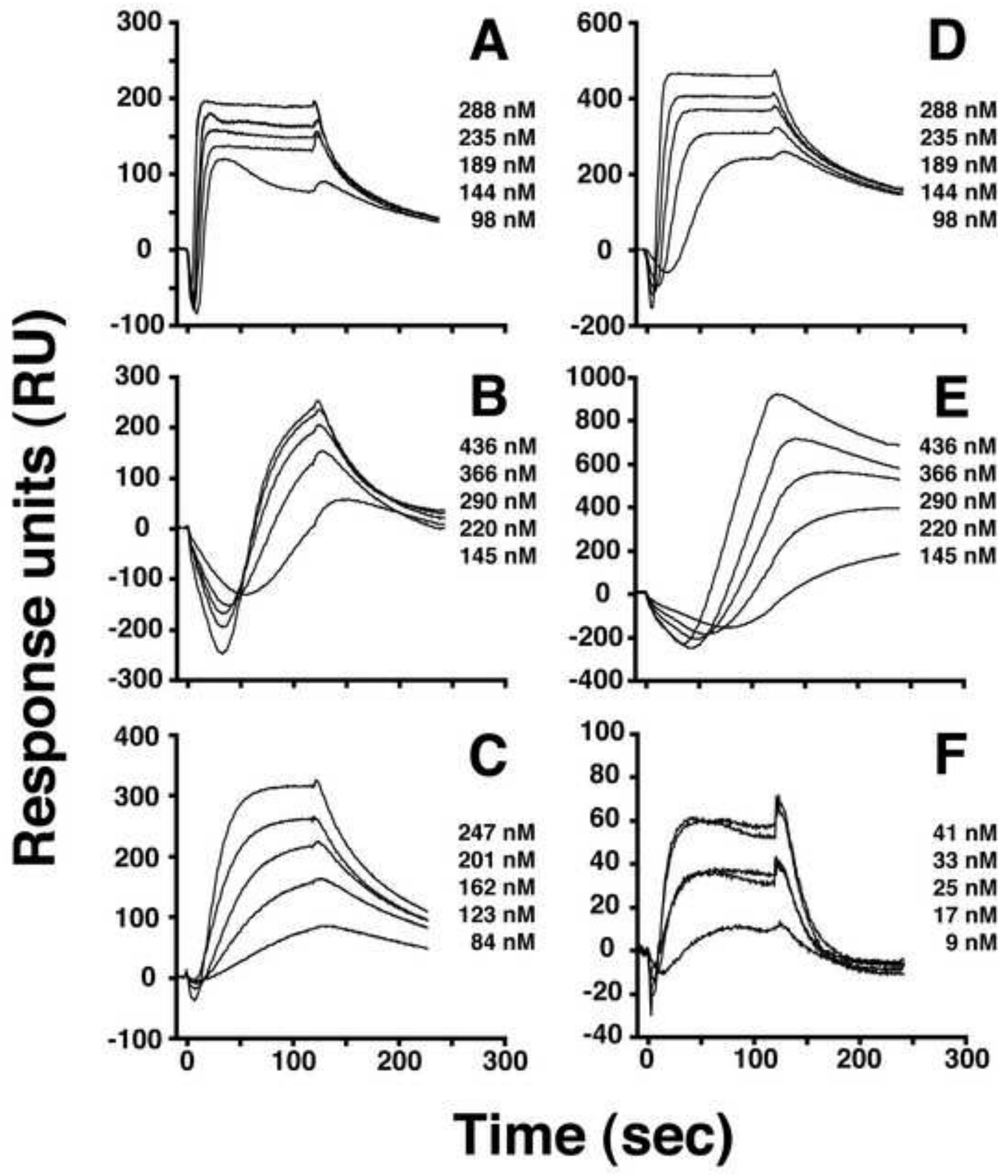


Table 1 Analysis of GAG composition of the fractions isolated from the FM-GAG fraction $^{a}$

\begin{tabular}{|c|c|c|}
\hline & $\begin{array}{l}\text { 1.0 M NaCl-eluted FM-GAG } \\
\text { fraction }\end{array}$ & $\begin{array}{l}\text { 2.0 M NaCl-eluted FM-GAG } \\
\text { fraction }\end{array}$ \\
\hline & \multicolumn{2}{|c|}{ pmol as disaccharides/mg tissue } \\
\hline $\mathrm{CS} / \mathrm{DS}$ & $684(37.5)^{b}$ & $149(56.0)$ \\
\hline HA & $1,085(59.4)$ & $104(39.1)$ \\
\hline $\mathrm{KS}$ & $\mathrm{ND}^{c}$ & $11(4.1)$ \\
\hline HS & $57(3.1)$ & $2(0.8)$ \\
\hline Total GAG & $1,826(100)$ & $266(100)$ \\
\hline \multicolumn{3}{|c|}{$\begin{array}{l}{ }^{a} \text { FM-GAG fractions were digested with a mixture of CSases ABC and AC-II, a mixture of } \\
\text { Hepases I and III, or keratanase II. Each digest was analyzed by anion-exchange HPLC } \\
\text { after } 2 \mathrm{AB} \text {-derivatization, and the GAG composition of each fraction was quantified based } \\
\text { on the fluorescent intensity of the derivatives (see "Materials and methods"). }\end{array}$} \\
\hline
\end{tabular}


Table 2 Disaccharide composition of the FM-CS/DS preparations ${ }^{a}$

\begin{tabular}{|c|c|c|c|c|c|c|}
\hline \multirow{2}{*}{$\mathrm{CS} / \mathrm{DS}$ disaccharides } & \multicolumn{3}{|c|}{ 1.0 M NaCl-eluted FM-CS/DS } & \multicolumn{3}{|c|}{ 2.0 M NaCl-eluted FM-CS/DS } \\
\hline & CSases $\mathrm{ABC}$ and $\mathrm{AC}-\mathrm{II}$ & CSases AC-I and AC-II & CSase B & CSases $\mathrm{ABC}$ and $\mathrm{AC}-\mathrm{II}$ & CSases AC-I and AC-II & CSase B \\
\hline & \multicolumn{3}{|c|}{ pmol as disaccharides/mg tissue } & \multicolumn{3}{|c|}{ pmol as disaccharides/mg tissue } \\
\hline$\Delta$ HexA-GalNAc & $277.1(40.5)^{b}$ & $250.6(45.9)$ & ND & $14.8(9.9)$ & $12.2(16.9)$ & ND \\
\hline$\Delta$ HexA-GalNAc(6S) & $178.9(26.2)$ & $166.1(30.3)$ & ND & $41.1(27.6)$ & $35.1(48.2)$ & ND \\
\hline$\Delta$ HexA-GalNAc(4S) & $223.5(32.7)$ & $130.3(23.8)$ & $17.3(97.4)$ & $90.9(60.9)$ & $25.3(34.8)$ & $12.2(94.8)$ \\
\hline$\Delta \operatorname{HexA}(2 \mathrm{~S})-\mathrm{GalNAc}(6 \mathrm{~S})$ & $2.6(0.4)$ & $\mathrm{ND}^{c}$ & ND & $0.7(0.5)$ & ND & ND \\
\hline$\Delta \operatorname{HexA}(2 \mathrm{~S})$-GalNAc(4S) & $1.4(0.2)$ & ND & $0.5(2.6)$ & $1.4(0.9)$ & ND & $0.7(5.2)$ \\
\hline$\Delta$ HexA-GalNAc(4S,6S) & $0.2(0.03)$ & ND & ND & $0.2(0.2)$ & $0.1(0.1)$ & ND \\
\hline Total & $683.7(100)$ & $546.9(100)$ & $17.7(100)$ & $149.1(100)$ & $72.7(100)$ & $12.9(100)$ \\
\hline $\mathrm{S} /$ unit $^{d}$ & 0.6 & 0.5 & 1 & 0.9 & 0.8 & 1.1 \\
\hline
\end{tabular}


Table 3 Kinetic parameters for the interactions of neurotrophic factors with immobilized FM-CS/DS preparations ${ }^{a}$

\begin{tabular}{ccccccc}
\hline \multirow{2}{*}{$\begin{array}{c}\text { Neurotrophic } \\
\text { factors }\end{array}$} & \multicolumn{3}{c}{$1.0 \mathrm{M} \mathrm{NaCl}$-eluted FM-CS/DS } & \multicolumn{4}{c}{2.0 M NaCl-eluted FM-CS/DS } \\
\cline { 2 - 7 } & $k_{a}$ & $k_{d}$ & $K_{d}$ & $k_{a}$ & $k_{d}$ & $S_{d}$ \\
\hline$M^{-1} s^{-1}$ & $s^{-1}$ & $n M$ & $M^{-1} s^{-1}$ & $(1.9 \pm 0.8) \times 10^{4}$ & $(8.5 \pm 4.2) \times 10^{-3}$ & $463.4 \pm 245.1$ \\
PTN & $-^{b}$ & - & - & $(1.2 \pm 0.5) \times 10^{6}$ & $(6.0 \pm 2.4) \times 10^{-2}$ & $53.3 \pm 16.1$ \\
\hline
\end{tabular}

${ }^{a}$ The $k_{a}, k_{d}$, and $K_{d}$ values were determined using a 1:1 binding model with mass transfer as described under "Materials and methods". The values for each neurotrophic factor are expressed as the mean \pm S.E. obtained with five different concentrations.

${ }^{b}$-, no significant interaction with the $1.0 \mathrm{M} \mathrm{NaCl}$-eluted FM-CS/DS preparation. 